Annales Geophysicae (2003) 21: 1095-1100 (c) European Geosciences Union 2003

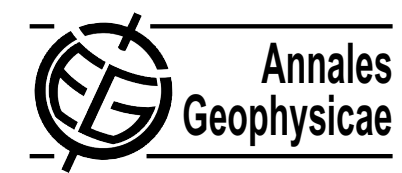

\title{
An investigation into the correlation of geomagnetic storms with tropospheric parameters over the South Pole
}

\author{
M. M. Lam and A. S. Rodger \\ British Antarctic Survey, Natural Environment Research Council, High Cross, Madingley Road,Cambridge CB3 0ET, UK
}

Received: 10 January 2002 - Revised: 21 November 2002 - Accepted: 26 November 2002

\begin{abstract}
We test the proposal that the Sun's magnetic activity, communicated via the solar wind, provides a link between solar variability and the Earth's climate in the Antarctic troposphere. The strength of a geomagnetic storm is one indicator of the state of the solar wind; therefore, we use the dates of 51 moderate to strong winter geomagnetic storms from the period 1961-1990 to conduct a series of superposed epoch analyses of the winter South Pole isobaric height and temperature, at pressures of between 100-500 mbar. Using Student's $t$-test to compare the mean value of the pre- and post-storm data sets, we find no evidence to support the hypothesis that there is a statistically-significant correlation between the onset of a geomagnetic storm and changes in the isobaric temperature or height of the troposphere and lower stratopshere over the South Pole during winter months. This concurs with a similar study of the variability of the troposphere and lower stratosphere over the South Pole (Lam and Rodger, 2002) which uses drops in the level of observed galactic cosmic ray intensity, known as Forbush decreases, as a proxy for solar magnetic activity instead of geomagnetic storms.
\end{abstract}

Key words. Interplanetary physics (solar wind plasma; cosmic rays) - Atmospheric composition and structure (pressure, density and temperature)

\section{Introduction}

The variability of the Sun and of the Earth's climate are well established, but the extent to which the two are closely linked is not clear. What is missing, at present, is a complete understanding of the various physical mechanisms that connect the Sun to the Earth's climate. Many hypotheses concentrate on the link between the Sun's output of electromagnetic radiation and the climate on Earth (e.g. Lean et al., 1995; White et al., 1997; Haigh, 1999), but there have been proposals that the Sun's magnetic activity, communicated via the

Correspondence to: M. M. Lam (M.Lam@ bas.ac.uk) solar wind, is a significant factor (Tinsley and Deen, 1991; Svensmark and Friis-Christensen, 1997; Soon et al., 2000; Lockwood, 2001; Stamper et al., 1999). If this proves to be the case, then there are, at present, three main contenders for the possible mechanism involved: first, Svensmark and Friis-Christensen (1997) propose that the ionizing effect of galactic cosmic rays influences climate-changing cloud formation and cover, by increasing the number of cloud condensation nuclei (Svensmark and Friis-Christensen, 1997; Marsh and Svensmark, 2000). The Sun's magnetic field modulates the flux of cosmic rays impinging on the Earth's atmosphere, forming a link between solar variability and climate variability. A second theory is that the solar wind can modulate the vertical atmospheric air-Earth current density on a variety of time scales (Tinsley, 1996). The resulting electrical effects on cloud microphysics (electro-freezing) give rise to changes in the dynamics of the atmosphere. Thirdly, Arnold and Robinson (1998) postulate that the deposition, due to geomagnetic activity, of energy via particle precipitation and Joule heating into the lower thermosphere gives rise to changes in wind and temperature profiles there. These, in turn, affect the reflection and absorption of planetary and gravity waves. They have demonstrated that solar-cycle variations in the temperature and winds of the lower thermosphere can cause substantial variations in stratospheric temperatures. Their model did not extend into the troposphere; however, their work opens up the possibility that solar activity on a variety of scales may affect the stratosphere and possibly the troposphere via this mechanism.

Egorova et al. (2000) use cases studies and superposed epoch methods to analyse daily pressure, temperature and wind data above the Antarctic station Vostok $\left(78.5^{\circ} \mathrm{S}\right.$, $\left.106.9^{\circ} \mathrm{E}\right)$. They conclude that their data provide evidence that, within a day, Forbush decreases are followed by a daylong warming of about $10^{\circ} \mathrm{C}$ at an altitude of $6-7 \mathrm{~km}$. They also suggest that Forbush decreases are associated with a reduction in atmospheric pressure at all altitudes below $20 \mathrm{~km}$, and a reconstruction of the whole wind system which occurs between $0-5$ days after the Forbush decrease. With a view to 

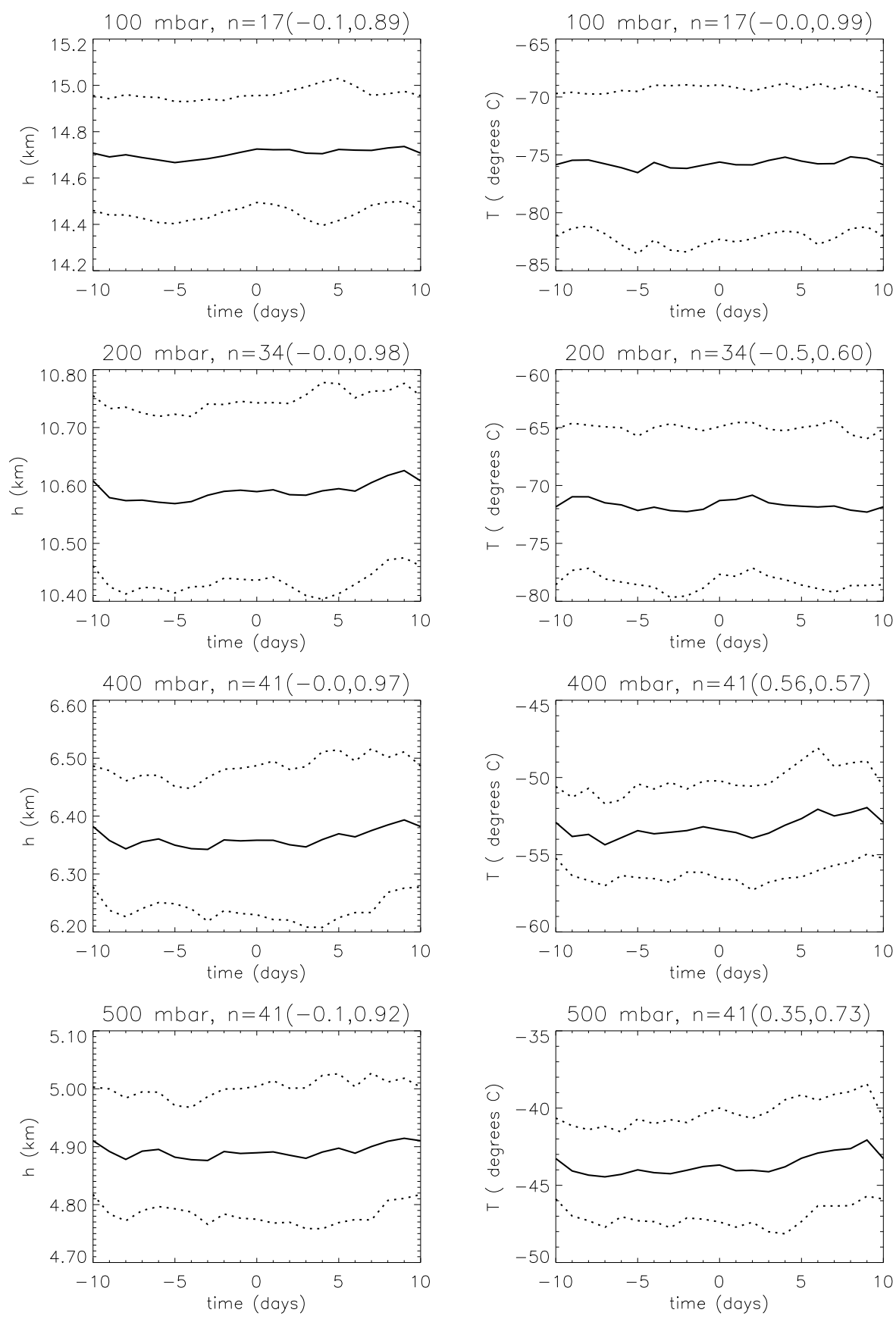

Fig. 1. Superposed epoch analyses of the winter isobaric height and temperature, at pressures of $100 \mathrm{mbar}, 200 \mathrm{mbar}, 400 \mathrm{mbar}$ and $500 \mathrm{mbar}$, with those for isobaric height in the left-hand column and those for isobaric temperature in the right-hand column. $t=0$ is the start time for each of $n$ geomagnetic storm events that meet the data selection criteria. The solid line in each plot represents the superposed epoch analysis and the dashed lines have been placed a standard deviation above and below the resulting superposition. The title of each plot gives the pressure level, the number of events used in the superposition and, in brackets, the $t$-test statistic and its significance for the difference between the mean values on days -1 and +1 .

finding the spatial extent of the Egorova et al. (2000) result, Lam and Rodger (2002) conducted a similar study using winter tropospheric isobaric temperature and height data at the South Pole $\left(0^{\circ} \mathrm{S}\right)$, located $1000 \mathrm{~km}$ away from Vostok. Using both the Egorova et al. (2000) set of Forbush decrease events (including the distinction of those with and without accom- panying solar-proton events), as well as their own set of Forbush decrease events, Lam and Rodger (2002) conclude that there are no observable changes in isobaric temperature and height associated with Forbush decreases. Having discussed the Egorova et al. (2000) method with one of the authors, and having investigated the Vostok data, Lam and Rodger (2002) 
consider that the difference between their results and those of Egorova et al. (2000) are due to significant differences in method.

Geomagnetic storms can be used as an indicator of changes in magnetic solar activity, in a similar way to Forbush decreases. Although Forbush decreases are often related to geomagnetic storms, we extend our original study (Lam and Rodger, 2002) by explicitly investigating whether any correlation can be found between moderate to strong geomagnetic storms and winter tropospheric isobaric temperature and height over the South Pole. An effect on climate is looked for without distinguishing between any of the three mechanisms mentioned above. Geomagnetic storms are initiated when there is an enhanced energy transfer from the solar wind/interplanetary magnetic field (IMF) into the magnetosphere. The $D_{s t}$ index is one well-accepted measure of the resulting intensification of the ring current (Tsurutani et al., 1999). In the initial phase of a geomagnetic storm, $D_{s t}$ typically increases to tens of $\mathrm{nT}$ for an interval lasting between minutes and h. Then $D_{s t}$ can drop to below hundreds of nT for up to several hours during the main phase followed by a gradual return to normal over a period of a day to a week (the recovery phase).

\section{Method and results}

The aim of this study is to ascertain whether any significant response to geomagnetic storms can be seen in the temperature or isobaric height of the atmosphere in the $12 \mathrm{~km}$ or so above the South Pole. Our principal method is to perform a superposed epoch analysis of the meteorological data with the start date of the storm defined as day 0 . We make use of upper air meteorological data from the National Oceanic and Atmospheric Administration/Climate Monitoring and Diagnostic Laboratory (NOAA/CMDL) observatory at South Pole, available at the ftp://ftp.cmdl.noaa/gov/met/ raobs/ website. The instruments used to measure the temperature and pressure data are a platinum resistance probe and a pressure transducer. We use data in the period 1961-1990. As is the case for Egorova et al. (2000), only the meteorological data during May-September are used, in order to exclude the influence of direct solar radiation.

In order to get the best chance of seeing a response in our superposed epoch analysis of data, we use moderate to strong geomagnetic storms which appear at otherwise fairly quiet times. Our method selects storms which are preceded by a period of non-storm activity and which possess a well-defined onset and a steady, roughly monotonic decay. The purpose of this is to ensure that any stormrelated effects on the South Pole meteorology appear in a consistent fashion, as there may be a delay of greater than one day between the storm onset and its effect in the atmosphere. We first place the hourly $D_{s t}$ index, available from the National Oceanic and Atmospheric Administration/National Geophysical Data Center (NOAA/NGDC) website at http:/www.ngdc.noaa.gov/, into 12-hourly bins.
We identify the start of a storm as the time when the binned value of $D_{s t}$ data first falls below $-70 \mathrm{nT}$. If the $D_{s t}$ index $m$ half-days later is larger in magnitude by a factor of $(1.25)^{\mathrm{m}}$, then the first date is ignored and the later one is used. This provides a consistent and objective way of choosing the time of the beginning of a storm when a storm takes more than $12 \mathrm{~h}$ to reach its minimum value, and is especially useful for identifying the start of very strong storms. If the storm amplitude decays away during the next 5 days, then the date is accepted; however, if another significant minimum (of less than $-70 \mathrm{nT}$ and at least half the size of the first minimum) appears within this time, then the date is discarded. Also, if there is any significant storm activity in the 5 days leading up to a storm date, then that date is discarded. Our results will be limited by the assumption (which we consider to be reasonable) that any reaction of the atmosphere to the presence of a geomagnetic storm occurs within a period of 5 days. The 51 dates resulting from our analysis are presented in Table 1.

Using the dates in Table 1, we conduct a superposed epoch analysis of the winter isobaric height and temperature, at pressures of $100 \mathrm{mbar}, 200 \mathrm{mbar}, 400 \mathrm{mbar}$ and $500 \mathrm{mbar}$. We discard events for which there is a gap in the data of more than $48 \mathrm{~h}$ in the period of the analysis, which commences 10 days before the date of a geomagnetic storm and ends 10 days after that date. The results are presented in Fig. 1, with those for isobaric height in the left-hand column and those for isobaric temperature in the right-hand column. The solid line in each plot represents the superposed epoch analysis and the dashed lines have been placed a standard deviation above and below the resulting superposition. The title of each plot gives the pressure level, the number of events used in the superposition and, in brackets, the $t$-statistic and its significance for the difference between the mean values on days -1 and +1 .

A very small difference between the means of the isobaric height on days -1 and +1 of a superposed epoch study could be significant if the number of data points is large. Conversely, a difference may be large yet not significant if there are few data points. Student's $t$-test uses the concept of "standard error" to measure the significance of a difference in the means of two data sets. Typically the standard error is equal to the sample's standard deviation divided by the square root of the number of points in the sample. When two distributions are thought to have the same variance but possibly different means, then the $t$-statistic for sample populations $x$ and $y$, with means $\bar{x}$ and $\bar{y}$, is computed by estimating the standard error of the difference of means from the pooled variance using the formula:

$t=\frac{\bar{x}-\bar{y}}{S . E}$,

where S.E is the standard error and is equal to:

$\sqrt{\frac{\left(N^{-1}+M^{-1}\right)\left[\sum_{i=0}^{N-1}\left(x_{i}-\bar{x}\right)^{2}+\sum_{i=0}^{M-1}\left(y_{i}-\bar{y}\right)^{2}\right]}{N+M-2}}$,

where $N$ and $M$ are the number of points in the first and second samples, respectively. The Student's distribution proba- 
bility function (not given here), $\mathrm{A}(t \mid v)$, gives the probability that two distributions with equal means would not generate a $t$-statistic this large just by chance. Two means are considered to be significantly different if the Student's distribution probability function is greater than 0.99 . The significance level is equal to $1-\mathrm{A}(t \mid v)$; therefore, a small numerical value of the significance (about 0.01 to 0.05 ) means that the observed difference is very significant. We use the IDL routine TM-test to compute Student's $t$-test statistic and significance using the data from days -1 and +1 , to determine if there is a significant difference between the pre- and post-storm means of the isobaric height (or temperature). We make the assumption that the variance of the data sets on days -1 and +1 are equal; the average percentage difference between the variances of the day -1 and day +1 data sets is $4 \%$ for the 8 cases presented in Fig. 1, which we deem to be acceptable for use with the Student's $t$-test. As the significance is never below 0.5 in value, we conclude that the onset of moderate to strong geomagnetic storms is not associated with a statistically-significant change in the winter tropospheric isobaric temperature or height over the South Pole.

Figure 4 of Egorova et al. (2000) is a scatter plot which compares the daily values of the winter temperature and pressure over Vostok, with the temperature and pressure the day after a set of Forbush decrease events. It is a simple but visually-striking plot and seems to present a clear indication that Forbush decreases are associated with a change in the value of winter tropospheric temperature and pressure. In Fig. 2 of Lam and Rodger (2002), the South Pole winter tropospheric data are presented in an almost identical fashion, but show no indication of a correlation between the Lam and Rodger (2002) set of Forbush decrease events and noticeable changes in the values of upper air data. No statistical method, however, is used to confirm this result.

To allow for a comparison with the studies of Egorova et al. (2000) and Lam and Rodger (2002), we also present our data in the form of a scatter plot, but we increase the rigour of the method by normalising the data first and conducting a Student's $t$-test to compare the mean of all daily values with the mean of the values which occur the day after our set of geomagnetic storms, that is, for $\Delta t=1$. We normalise the data with the mean of the values commencing 15 days before a given data point and ending 15 days after it, which we refer to as the running-monthly-mean for convenience. In the plots on the left-hand side of Fig. 2, each spot represents the difference between the isobaric height, $h$, and the running-monthly-mean isobaric height, $h_{m}$, as a percentage of the running-monthly-mean height for the day after a geomagnetic storm $(\Delta t=1)$ :

$h($ spot $)=100 \frac{\left(h(\Delta t=1)-h_{m}(\Delta t=1)\right)}{h_{m}(\Delta t=1)}$.

The results for isobaric temperature are presented in a similar fashion in the right-hand column. The title of each plot gives the pressure level and the number of storm dates (the number of spots), $n$, with good data. The solid line in each of the plots shows the mean (over all years of data used) of the
Table 1. Dates of 51 moderate to strong winter geomagnetic storms in the period 1961-1990, as identified from $D_{s t}$ data

\begin{tabular}{rrr}
\hline 28 Jul. 1961 & 15 Sep. 1963 & 23 Sep. 1963 \\
27 May 1966 & 4 Sep. 1966 & 3 May 1967 \\
26 May 1967 & 6 Jun. 1967 & 21 Sep. 1967 \\
29 Sep. 1967 & 8 Sep. 1968 & 15 May 1969 \\
30 Sep. 1969 & 29 May 1970 & 26 Jul. 1970 \\
17 Aug. 1970 & 18 Jun. 1972 & 14 Sep. 1972 \\
14 May 1973 & 6 Jul. 1974 & 16 Sep. 1974 \\
3 May 1976 & 10 May 1978 & 5 Jul. 1978 \\
28 Aug. 1978 & 30 Sep. 1978 & 30 Aug. 1979 \\
19 Sep. 1979 & 26 May 1980 & 11 May 1981 \\
26 Jul. 1981 & 24 Aug. 1981 & 27 Sep. 1981 \\
14 Jul. 1982 & 7 Aug. 1982 & 7 Sep. 1982 \\
14 Jun. 1983 & 8 Aug. 1983 & 5 Sep. 1984 \\
12 Sep. 1986 & 29 Jul. 1987 & 26 Aug. 1987 \\
7 May 1988 & 12 Sep. 1988 & 25 May 1989 \\
11 Jun. 1989 & 15 Aug. 1989 & 29 Aug. 1989 \\
27 Sep. 1989 & 13 Jun. 1990 & 29 Jul. 1990 \\
\hline
\end{tabular}

difference between the daily value and the running-monthlymean value, as a percentage of the running-monthly-mean value. The dashed lines have been placed a standard deviation above and below the mean. The numbers in brackets within each plot give the $t$-statistic and its significance for the difference between the means of the complete winter data set and the data set associated with geomagnetic storms. Again, we make the assumption that the variance of the two data sets under comparison are equal (the average value of the difference between the variances of the two data sets concerned for the 8 cases presented in Fig. 2 is $8 \%$ ). There are no statistically-significant differences between the means of the two data sets, indicating that we are again unable to find a correlation between the onset of moderate to strong geomagnetic storms and changes in winter tropospheric isobaric temperature or height over the South Pole.

We have also conducted Student's $t$-test to compare the mean of all daily values with the mean of the storm-related values for $\Delta t=$ (a) -2 days, (b) -1 day, (c) 0 days, (d) 2 days, (e) 3 days and (f) 4 days, that is, we looked for a significant correlation between the date of storm onsets and variability in the lower atmosphere every day in the range -2 to 4 days after a storm onset. No plots are presented of these results. Again, no statistically-significant differences were found between the mean of the complete winter data set and the mean of the data set associated with geomagnetic storms.

\section{Conclusions}

Using superposed epoch analyses of upper air data around the time of moderate to strong winter geomagnetic storms, and utilising Student's $t$-test to examine the difference between the means of the superposition on days -1 and +1 , we conclude that no statistically-significant correlation can 

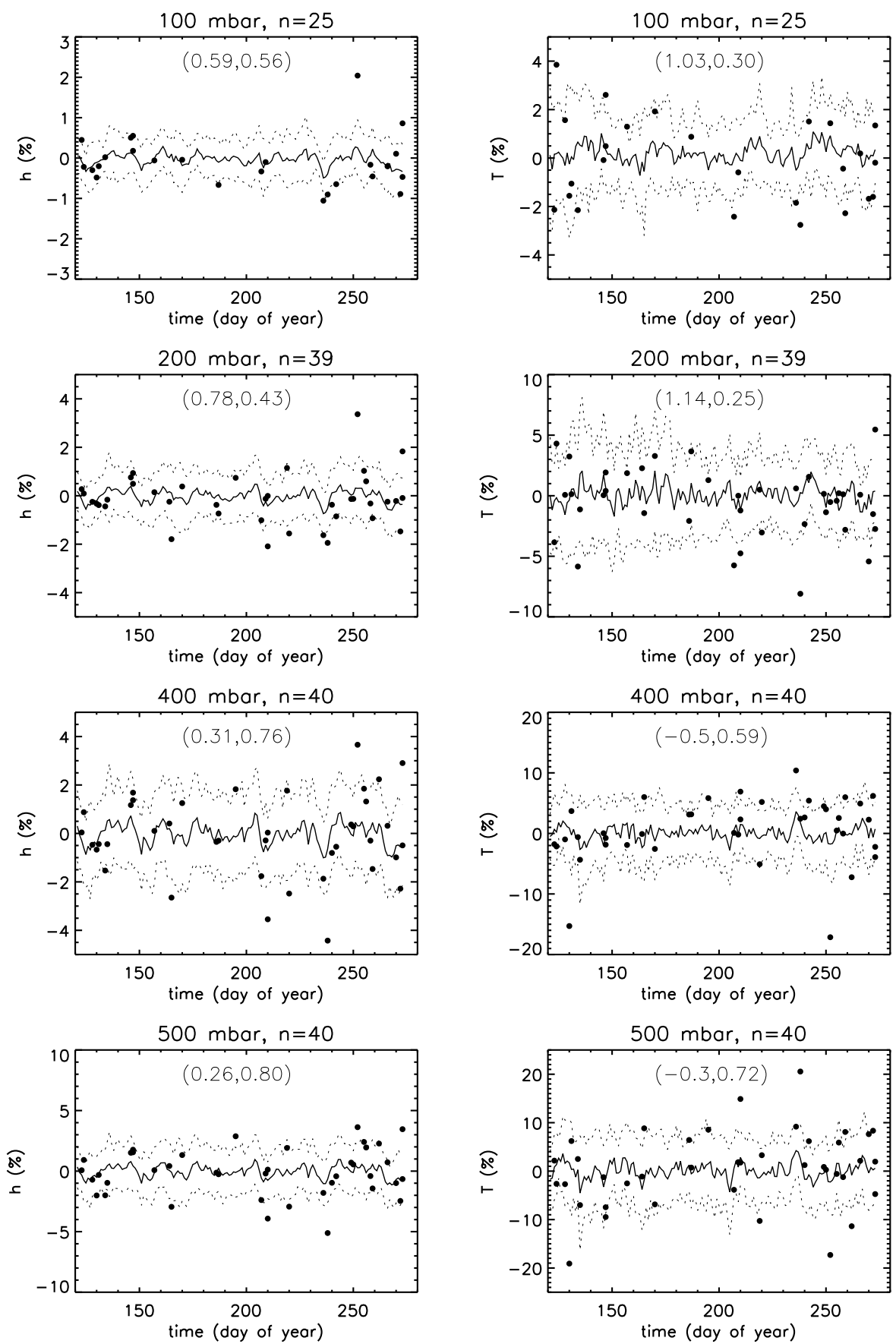

Fig. 2. South Pole winter meteorological data in the form of scatter plots, with those relating to isobaric height in the left-hand column and to isobaric temperature in the right-hand column. The title of each plot gives the pressure level and the number of storm dates (number of spots), $n$, with good data. The solid line shows the mean (over all years of data used) of the difference between the daily value and the running-monthly-mean value, as a percentage of the running-monthly-mean value. The dashed lines have been placed a standard deviation above and below the mean. The numbers in brackets within each plot give the $t$-test statistic and its significance for the difference between the means of the complete winter data set and the data set associated with geomagnetic storms.

be found between the occurrence of such geomagnetic storms and any variation in value of the isobaric temperature or isobaric height of the troposphere and lower stratosphere over the South Pole during winter months. This is in accord with our previous study, which utilised winter Forbush decreases as an indicator of solar variability, on the same tropospheric and lower stratospheric parameters over the South Pole (Lam and Rodger, 2002). In addition, our analysis suggests that the mechanism proposed by Arnold and Robinson (1998) does not vary lower stratospheric parameters over the South Pole 
in winter, even though there is a significant quasi-stationary, planetary wave activity in the mesosphere (Smith, 1997) during the winter. One might not expect a contribution from gravity wave fluxes, which are probably low due to the lack of orographic features and severe weather systems over the Antarctic in winter (King and Turner, 1997).

Acknowledgements. We would like to thank NOAA for the provision of $D_{s t}$ data, and Tom Mefford at NOAA for his helpfulness with regard to the provision of South Pole meteorological data. We would also like to thank G. Chanteur of Annales Geophysicae and the referees of the original manuscript for their helpful comments which enabled us to make significant improvements to the paper.

Topical Editor G. Chanteur thanks E. Posmentier and C. Peymirat for their help in evaluating this paper.

\section{References}

Arnold, N. F. and Robinson, T. R.: Solar cycle changes to planetary wave propagation and their influence on the middle atmosphere circulation, Ann. Geophysicae, 16, 69-76, 1998.

Egorova, L. V., Vovk, V. Ya., and Troshichev, O. A.: Influence of variations of the cosmic rays on atmospheric pressure and temperature in the southern geomagnetic pole region, J. Atmos. Solar-Terr. Phys., 62, 955-966, 2000.

Haigh, J. D.: Modelling the impact of solar variability on climate, J. Atmos. Solar-Terr. Phys., 61, 63-72, 1999.

King, J. C. and Turner, J.: Antarctic Meteorology and Climatology, Cambridge University Press, 1997.

Lam, M. M. and Rodger, A. S.: The effect of Forbush decreases on the troposphere over South Pole, J. Atmos. Solar-Terr. Phys., 64, 41-45, 2002.
Lean, J., Beer, J., and Bradley, R.: Reconstruction of solar irradiance since 1610: implications for climate change, Geophys. Res. Lett., 22, 3195-3198, 1995.

Lockwood, M.: Long-term variations in the magnetic fields of the Sun and the heliosphere: their origins, effects and implications, J. Geophys. Res., 106, 16 021-16038, 2001.

Marsh, N. and Svensmark, H.: Cosmic rays, clouds and climate, Space Sci. Rev., 94, 215-230, 2000.

Smith, A. K.: Stationary planetary waves in upper mesospheric winds, J. Atmos. Sci., 54, 2129-2145, 1997.

Stamper, R., Lockwood, M., Wild, M. N., and Clark, T. D. G.: Solar causes of the long-term increase in geomagnetic activity, J. Geophys. Res., 104, 28 325-28 342, 1999.

Soon, W., Baliunas, S., Posmentier, E. S., and Okeke, P.: Variations of solar coronal hole area and terrestrial lower tropospheric air temperature from 1979 to mid-1998: astronomical forcings of change in Earth's climate?, New Astronomy, 4, 563-579, 2000.

Svensmark, H. and Friis-Christensen, E.: Variation of cosmic ray flux and global cloud coverage - a missing link in solar-climate relationships, J. Atmos. Solar-Terr. Phys., 59, 1225-1232, 1997.

Tinsley, B. A.: Correlations of atmospheric dynamics with solar wind-induced changes of air-earth current density into cloud tops, J. Geophys. Res., 101, 29 701-29714, 1996.

Tinsley, B. A. and Deen, G. W.: Apparent tropospheric response to $\mathrm{MeV}-\mathrm{GeV}$ particle flux variations: a connection via electrofreezing of supercooled water in high-level clouds?, J. Geophys. Res., 96, 22 283-22 296, 1991.

Tsurutani, B. T., Kamide, Y., Arballo, J. K., Gonzalez, W. D., and Lepping, R. P.: Interplanetary causes of great and superintense magnetic storms, Phys. Chem. Earth, 24, 101-105, 1999.

White, W. B., Lean, J., Cayan, D. R., and Dettinger, M. D.: Response of global upper ocean temperature to changing solar irradiance, J. Geophys. Res., 102, 3255-3266, 1997. 